\title{
Review: Proteomic approaches to control lactational parameters in dairy cows
}

\author{
P. D. Eckersall ${ }^{1,2 \dagger}$ \\ ${ }^{1}$ VetMedZg Laboratory, Faculty of Veterinary Medicine, University of Zagreb, Heinzelova 55, Zagreb, Croatia; ${ }^{2}$ Institute of Biodiversity, Animal Health and \\ Comparative Medicine, University of Glasgow, Glasgow, UK
}

(Received 22 August 2018; Accepted 27 November 2018)

The use of a proteomic approach to investigate changes in the milk proteome is growing and has parralleled the increasing technological developments in proteomics moving from early investigation using a gel-based two-dimensional separation approach to more quantitative method of current focus applying chromatography and mass spectrometry. Proteomic approaches to investigate lactational performance have made substantial findings especially in the alterations in lactation during mastitis. An experimental model of Streptococcus uberis infection of the mammary gland has been used as a means to determine change not only in the milk proteome, but also in the peptidome and in the metabolome caused by the infection. Examination of the peptidome, that is the peptides of less than $25 \mathrm{kDa}$ in molecular weight, demonstrated an increase in small peptides most of which were casein degradation products but also included small bioactive peptides such as mammary-associated serum amyloid A3 (MSAA3). The peptidome has also been shown to differ depending on the causative bacteria of naturally occuring mastitis. The use of a non-gel-based relative quantitative proteomic methodology has revealed major changes in the protein component of milk in mastitis. The S. uberis infection lead to increases in the concentrations of proteins such as cathelicidins, haptoglobin, MSAA3 and decreases milk content of proteins such as xanthine oxidase, butyrophilin and $\beta-1,4-$ galactosyltransferase. Analysis of all protein change data identified the acute phase, coagulation and complement pathways as well as proteins related to bile acid metabolism as being most modified. Examination of the small molecular weight organic molecules of milk using a metabolomic approach identified an increase in the content in milk during mastitis of bile acids such as taurochenodeoxycholic acid. Notable changes were also found in metabolites responding to infection of the mammary gland. Carbohydrate and nucleic acid metabolites were reduced, whereas lipid and nitrogen containing metabolites were increased. The latter included increases in amino acids along with di and tri peptides, likely to be the result of casein degradation. The use of proteomics and other omic technology is in its infancy in investigation of lactational parameters, but can already provide additional insight into the changes involved in disease and will have further value in physiological and nutritional investigation of lactation.

Keywords: milk, proteomics, mastitis, biomarkers, haptoglobin

\section{Implications}

The application of the advanced analytical technology of proteomics to the study of milk from dairy cows has been growing over the last few decades. This approach allows detection and measurement of a vast number of proteins in milk and has allowed studies to be undertaken of the changes that take place in milk, especially in response to infections of the mammary gland that cause the major disease of bovine mastitis. This has revealed major changes in the milk proteins with some protein such as haptoglobin ( $\mathrm{Hp}$ ) increasing in concentration while others such as $\alpha$-lactalbumin decreasing in following infection.

\footnotetext{
${ }^{\dagger}$ E-mail: david.eckersall@glasgow.ac.uk
}

\section{Proteomics in lactation biology}

Proteomics which is the application of advanced technology such as nano liquid chromatography (LC) and mass spectrometry (MS) to characterise the full complement of proteins in a biological sample, has had an increasing role in investigations of animal science and veterinary medicine (Almeida et al., 2014). This is not only to provide novel insights into the physiology and pathophysiology of farm and companion animals but also to provide insight into questions on basic scientific questions which includes the processes involved in lactation required for maximising milk production (Bilic et al., 2018). While still to mature into a widespread technology, proteomics and related omic approaches such as metabolomics, the analysis of all metabolites in a sample, there has 
been considerable progress. This is exemplified by contrasting the major texts on animal proteomic approaches separated by only seven years from 2011 (Eckersall and Whitfield, 2011) to 2018 (Almeida et al., 2018) showing evidence of an active scientific community developing this new discipline for investigation of animal biology in relation to livestock production in agriculture and in the health of companion animals. In relation to investigation of lactation, there have been reviews of application of proteomics in study of milk as a nutritious food and in its processing into cheeses (Soggiu et al., 2018) as well as applications to the study of mammary gland biology in health and disease (Almeida and Eckersall, 2018).

Investigation of mastitis and the changes in the proteome and the metabolome of milk is one of the major areas where these approaches have been applied and has yielded results of significant impact especially in the search for biomarkers in milk of mastitis, the major economically important disease of dairy (Mudaliar et al., 2017). This has implications for animal welfare and also for potentially reducing the use antimicrobials for treatments that are causing concern in relation to the growth of antimicrobial resistance of the relevant pathogens. This review will therefore focus on recent reports on the use of different approaches of using proteomic and other omic technologies to investigate this specific but important area of lactation biology in ruminants.

\section{Lactational change in mammary infection}

Interest in the use of proteomics in investigation of the change in milk protein in response to mastitis has evolved over time along with advances in proteomic technology. Earlier investigations made use of two-dimensional electrophoresis (Hogarth et al., 2004) but more recently the use of LC and MS (LC-MS/MS) has allowed improved quantification and increased the sensitivity of detection for less abundant proteins (Soggiu et al., 2018). These non-gel-based, quantitative proteomic methods are being increasingly applied to the measurement of multiple proteins in milk in either experimentally induced or naturally occurring mastitis.

A study of mastitis caused by Staphylococcus aureus infection in which milk whey from cows with this bacteria were compared to milk from healthy cows and used a labelled free quantitative approach to identify differentially abundant protein in the whey (Abdelmegid et al., 2018). In all, 90 proteins were identified, and 25 of these showed significant differences between groups. The overriding functional activity of these proteins was that they are involved in the host defence against infection largely as part of the innate immune system. The functions the proteins fulfil included antimicrobial activity, pathogen recognition and the acute phase response. Within the latter group, $\mathrm{Hp}$ and lipopolysaccharide-binding protein (LBP) were identified, whereas cathelicidin-4 (CATHL4), peptidoglycan recognition protein 1 (PGLYRP1), lactoperoxidase (LPO), lactotransferrin
(Lactoferrin) (LTF), histone proteins (histone $\mathrm{H} 4$ and histone $\mathrm{H} 2 \mathrm{~A}$ ) and cathepsin $B$ (CTSB) were also proteins that were increased in $S$. aureus infection. Proteins involved in pathogen-recognition also included LBP as well as monocyte differentiation antigen (CD14), and chitinase-3-like protein 1 (CHI3L1).

A quantitative proteomic approach has also been taken in the search for the optimal biomarker, or combination of biomarkers for bovine disease (Zoldan et al., 2017). Biomarkers found in milk for a range of diseases, including mastitis but also non-mammary disease, indicated that the milk $\mathrm{Hp}$ concentration had the highest values among potential biomarkers for sensitivity and specificity and these discriminatory capabilities were increased in combination with the lactoferrin or secretory component, of immunoglobulin $\mathrm{A}$. It was apparent that the milk concentrations of $\mathrm{Hp}$ were increased by extra mammary inflammatory diseases, though the levels of milk $\mathrm{Hp}$ found by immunoassay were appreciably higher when mastitis was the causative disease. In a separate but relevant quantitative proteomics investigation (Altena et al., 2016), 13 milk proteins, including CD9, $\gamma$-glutamyl transferase, lactoferrin and CD36, were found by nano-LC-MS/MS to be more abundant in dairy cows classified as being less resistant to natural diseases, which, as well as mastitis, included lameness, retained placenta, metritis, respiratory diseases and metabolic diseases such as ketosis. It appears that systemic, non-mammary disease does affect the milk proteome. Whether such changes are sufficient to allow an assay in milk to be used as a diagnostic for health assessment in dairy cows will require extensive validation and careful implementation.

In post-discovery phases of potential biomarkers for mastitis, the use of selective reaction monitoring MS to quantify targeted specific proteins has been reported (Kusebauch et al., 2018). In this method isotope-labelled reference peptides were generated by chemical synthesis for 13 host response proteins considered to be altered in abundance in milk due to mastitis: lactoferrin, $\alpha-2$ macroglobulin, $\alpha-1$ antitrypsin, $\mathrm{Hp}$, serum amyloid $\mathrm{A} 3, \mathrm{CD} 14$, calgranulin $\mathrm{B}$, calgranulin $C$, cathepsin $C$, vanin-1, galectin-1, galectin-3 and interleukin-8. These proteins were shown to be altered by lipopolysaccharide endotoxin from gram-negative Escherichia coli and also the proteoglycan from gram positive $S$. aureus. However, the relative changes in abundance differed between these bacteria and if this altered host response is confirmed in future investigation this proteomic procedure could be the basis for a differential test for the gram-negative and gram-positive bacteria as the cause of the mastitis.

The validation of biomarkers identified by proteomics is a key process in adoption in diagnosis. This has been illustrated by initial identification of cathelicidin as a marker for mastitis (Addis et al., 2013). Although the initial discovery was made with a proteomic approach in ovine mammary epithelial cells following $S$. uberis mastitis, subsequent investigation of bovine mastitis using an immunoassay with an antibody to synthetic pan-cathelicidin peptides has shown that the cathelicidin in milk is highly correlated to somatic 
cell count the currently favoured test for mastitis, in over 500 milk samples used in validation studies. Cathelicidin has also been identified as a biomarker in Streptococcus agalactiae caused cases of mastitis, although by use of the classic proteomic method of two-dimensional electrophoresis along with MS (Pongthaisong et al., 2016).

\section{Integrated omics in lactation}

Proteomics is one of a number of 'omic' sciences which when combined provide the tools for a systems biology research approach. In mammary gland biology there have been many genomic and transcriptomic investigations (Mudaliar et al., 2017) but the integration of other technology such as proteomics and metabolomics has been rare. However, a combined investigation of $S$. uberis mastitis in which proteomics, metabolomics and peptidomics has been undertaken (Mudaliar et al., 2016; Thomas et al., 2016a and 2016b). These technologies were applied to determine the changes taking place in the metabolites, peptides and proteins following infection of mammary glands with of $S$. uberis (Tassi et al. 2013) and in relation to the resultant inflammation and final resolution. Overall this 'Mastitomics' investigation analysed small molecules with a mass less than $1500 \mathrm{Da}$ (metabolomics), peptides and polypeptide fragments in the range of 380 to $6000 \mathrm{Da}$ (peptidomics) and proteins with masses ranging up to $3 \mathrm{MDa}$ (proteomics). The omic results were compared to mastitis outcome based on clinical signs, somatic cell counts and bacterial count and acute phase protein concentration in milk at six time points postchallenge. Omic analysis was performed by capillary electrophoresis-MS, LC-MS/MS and LC-MS for peptidomic, proteomic and targeted metabolomic data, respectively, and quantitative results of relative abundance to control samples were generated.

Peptidomics revealed 460 peptides, of which 77 peptides were able to classify pre- and post-infection time points (Thomas et al., 2016b). Most peptides were degradation products of casein proteolysis. Other small peptides identified were serum amyloid $A$ and glycosylation-dependent cell adhesion molecule 1 (GDCAM). Peptidomics revealed a reduction in the caseins, first at 36-h post-challenge and continuing even after the elimination of infection, and the alteration in abundance were likely to be caused by host or bacterial proteases. A previous peptidomic investigation of natural clinical mastitis (Mansor et al., 2013) using the same approach demonstrated the possibility of combining peptide analysed into biomarker panel for diagnosis of mastitis and also for differential diagnosis between gram-positive and gram-negative bacteria causing the infection. Further validation of such an approach will be needed before milk peptide panels could be used for disease diagnosis.

Mastitomics proteomics quantified 570 bovine proteins that changed in abundance over the course of the infection (Mudaliar et al., 2016). Of particular interest was that the proteomics analysis showed more than an $1000 \times$ increase in antimicrobial proteins such as peptidoglycan recognition protein 1 and the cathelicidins between 36 and $81 \mathrm{~h}$ postchallenge. Acute phase proteins Hp, MSAA3 and C-reactive protein (CRP), showed increased abundance by LC-MS/MS, with results validated by immunoassay, which showed similar patterns of their change in abundance. Thus the acute phase proteins in milk started increasing by $100 \times$ at $30-\mathrm{h}$ post-challenge and reached a peak at around $57 \mathrm{~h}$ post challenge. Interestingly, CRP does have a major acute phase reaction in bovine milk during mastitis (Thomas et al., 2016b) with undetectable levels being found in milk from a healthy mammary gland. In contrast, in cattle CRP is present in serum from healthy animals and at best is a mild to moderate acute phase protein, rising only threefold during infection (Lee et al., 2003) and has even been reported as a negative acute phase response (Schroedl et al., 2014).

Metabolomics analysis putatively identified 690 metabolites with a particularly striking increase in the concentration of bile acids following infection (Thomas et al., 2016a). The bile acids that showed elevated abundance included glycocholate (C26H43NO6), taurocholic acid (C26H45NO7S), taurochenodeoxycholic acid (C26H45NO6S), glycodeoxycholate (C26H43NO5) and cholate (C24H4005). There was also a general increase in nitrogen containing metabolites, especially of free amino acid and di-tri-peptides, likely to be from casein degradation and also an increase in lipid metabolites. Carbohydrates and nucleic acids were in general decreased in milk by mastitis. The use of metabolomics to analyse milk during mastitis and provide potential biomarkers for the disease has also been reported (Dervishi et al., 2016), who concluded that the amino acids, valine, serine, tyrosine and phenylalanine were predictive for subacute mastitis, even when estimated by metabolomics 4 or 8 weeks before calving.

Integrating the analysis of the omics data particularly highlighted the up-regulation of bile acids in proteomic as well as metabolomic outcomes as pathways identified as upregulated by proteomics included liver $X$ receptor $(L X R)$, retinoid $\mathrm{X}$ receptor $(R X R)$ and Farnesoid $\mathrm{X}$ receptor $(F X R)$ activation pathways which are related to bile acid metabolism. The acute phase response signalling pathway was also shown to be the most up regulated by mastitis. An overview of the integrated analysis showed that while the bacterial count reached a maximum at 36 to $42 \mathrm{~h}$ post-challenge, most of the host responses by proteomics or metabolomics did not reach a maximum until 57 or $81 \mathrm{~h}$ post-challenge. The combination of omic approaches provided a mass of data for bioinformatic investigation and showed the potential for an integrative approach of advanced technology to give as complete a picture of the mammary gland's response to mastitis as is possible at present.

\section{Acknowledgements}

Colleagues at the School of Veterinary Medicine and Institute of Biodiversity, Animal Health and Comparative Medicine, University of Glasgow, UK and the VetMedZg Laboratory of the 
Faculty of Veterinary Medicine, University of Zagreb, Croatia for their contributions to the use of omic research in understanding the lactational response in mastitis. The BBSRC is thanked for the funding support for this area of research from Follow on Fund BB/M022021/1.

\section{Declaration of interest}

The author declares no conflicts of interest.

\section{Ethics statement}

Ethics approval reported in the original publications cited in this review.

\section{Software and data repository resources}

Where appropriate software and data repository resources are reported in the original publications cited in this review.

\section{References}

Abdelmegid S, Murugaiyan J, Abo-Ismail M, Caswell JL, Kelton D and Kirby GM 2018. Identification of host defense-related proteins using label-free quantitative proteomic analysis of milk whey from cows with staphylococcus aureus subclinical mastitis. International Journal of Molecular Science 19, 78.

Addis MF, Pisanu S, Marogna G, Cubeddu T, Pagnozzi D, Cacciotto C, Campesi F, Schianchi G, Rocca S and Uzzau S 2013. Production and release of antimicrobial and immune defense proteins by mammary epithelial cells following Streptococcus uberis infection of sheep. Infection and Immunity 81, 3182-3197.

Almeida AM, Bassols A, Bendixen E, Bhide M, Ceciliani F, Cristobal S, Eckersall PD, Hollung K, Lisacek F, Mazzucchelli G, McLaughlin M, Miller I, Nally JE, Plowman J, Renaut J, Rodrigues P, Roncada P, Staric J and Turk R 2014. Animal board invited review: advances in proteomics for animal and food sciences Animal 9, 1-17.

Almeida AM and Eckersall PD 2018. Proteomics and mammary gland research in dairy species. In Proteomics in domestic animals (ed. Almeida, AM, Eckersall, D and Miller, I), pp. 255-280. Springer International Publishing, Cham, Switzerland. Almeida AM, Eckersall D and Miller I 2018. Proteomics in domestic animals: from farm to systems biology. Springer International Publishing, Cham, Switzerland.

Altena SEC V, De Klerk B, Hettinga KA, Van Neerven RJJ, Boeren S, Savelkoul HFJ and Tijhaar EJ 2016. A proteomics-based identification of putative biomarkers for disease in bovine milk. Veterinary Immunology and Immunopathology 174 11-18.

Bilic P, Kules J, Galan A, de Pontes LG, Guillemin N, Horvatic A, Sabes AF, Mrljak V and Eckersall PD 2018. Proteomics in veterinary medicine and animal science: neglected scientific opportunities with immediate impact. Proteomics 47, 1-7.

Dervishi E, Zhang G, Hailemariam D, Dunn SM and Ametaj BN 2015. Innate immunity and carbohydrate metabolism alterations precede occurrence of subclinical mastitis in transition dairy cows. Journal of Animal Science and Technology $57,46$.
Eckersall PD and Whitfield PD 2011. Methods in animal proteomics. WileyBlackwell, London, UK.

Hogarth CJ, Fitzpatrick JL, Nolan AM, Young FJ, Pitt A and Eckersall PD 2004. Differential protein composition of bovine whey: a comparison of whey from healthy animals and from those with clinical mastitis. Proteomics 4, 2094-2100.

Kusebauch ULE, Hernández-Castellano TE, Bislev SL, Moritz RL, Røntved CM and Bendixen E 2018. Selected reaction monitoring mass spectrometry of mastitis milk reveals pathogen-specific regulation of bovine host response proteins. Journal of Dairy Science 101, 6532-6541.

Lee W-C, Hsiao H-C, Wu Y-L, Lin J-H, Lee Y-P, Fung H-P, Chen H-H, Chen Y-H and Chu R-M 2003. Serum C-reactive protein in dairy herds. Canadian Journal of Veterinary Research 67, 102-107.

Mansor R, Mullen W, Albalat A, Zerefos P, Mischak H, Barrett DC, Biggs A and Eckersall PD 2013. A peptidomic approach to biomarker discovery for bovine mastitis. Journal of Proteomics 85, 89-98.

Mudaliar M, Tassi R, Thomas FC, McNeilly TN, Weidt SK, McLaughlin M, Wilson D, Burchmore R, Herzyk P, Eckersall PD and Zadoks RN 2016. Mastitomics, the integrated omics of bovine milk in an experimental model of: Streptococcus uberis mastitis: 2. Label-free relative quantitative proteomics. Molecular Biosystems 12, 2748-2761.

Mudaliar M, Thomas FC and Eckersall PD 2017. Omic approaches to a better understanding of mastitis in dairy cows. In Periparturient diseases of dairy cows (ed. Ametaj, BN), pp. 139-182. Springer International Publishing, Cham, Switzerland.

Pongthaisong P, Katawatin S and Thamrongyoswittayakul C 2016. Milk protein profiles in response to Streptococcus agalactiae subclinical mastitis in dairy cows. Animal Science Journal 87, 92-98.

Schroedl W, Kleessen B, Jaekel L, Shehata AA and Krueger M 2014. Influence of the gut microbiota on blood acute-phase proteins. Scandinavian Journal of Immunology 79, 299-304.

Soggiu A, Poncada P and Piras C 2018. Proteomics in milk and dairy products. In Proteomics in domestic animals: from farm to systems biology (ed. Almeida, AM, Eckersall, PD and Miller, I), pp. 169-194. Springer International Publishing, Cham, Switzerland.

Tassi R, McNeilly TN, Fitzpatrick JL, Fontaine MC, Reddick D, Ramage C, Lutton M, Schukken YH and Zadoks RN 2013. Strain-specific pathogenicity of putative host-adapted and nonadapted strains of Streptococcus uberis in dairy cattle. Journal of Dairy Science 96, 5129-5145.

Thomas FC, Mudaliar M, Tassi R, McNeilly TN, Burchmore R, Burgess K, Herzyk P, Zadoks RN and Eckersall PD 2016a. Mastitomics, the integrated omics of bovine milk in an experimental model of: Streptococcus uberis mastitis: 3 . Untargeted metabolomics. Molecular Biosystems 12, 2762-2769.

Thomas FC, Mullen W, Tassi R, Ramírez-Torres A, Mudaliar M, McNeilly TN, Zadoks RN, Burchmore R and Eckersall PD. 2016b. Mastitomics, the integrated omics of bovine milk in an experimental model of Streptococcus uberis mastitis: 1. High abundance proteins, acute phase proteins and peptidomics. Molecular BioSystems 12, 2735-2747.

Zoldan K, Schneider J, Moellmer T, Fueldner C, Knauer J, Fuerll M, Starke A, Specht M, Reiche K, Hackermueller Kalkhof JS and Bergen V 2017. Advances in dairy research discovery and validation of immunological biomarkers in milk for health monitoring of dairy cows - results from a multiomics approach. Advances in Dairy Research 5, 3. 